Article

\title{
Covid-19 Vaccine Passport and International Traveling: The Combined Effect of Two Nudges on Americans' Support for the Pass
}

\author{
Chiara Sotis ${ }^{1}$, Miriam Allena ${ }^{2}$, Renny Reyes and Alessandro Romano ${ }^{2,4 *}$ \\ 1 London School of Economics \\ 2 Bocconi University \\ 3 Pontificia Universidad Católica Madre y Maestra \\ 4 Yale University \\ * Correspondence: c.sotis@lse.ac.uk
}

Citation: Sotis, C.; Allena, M.;

Reyes, R.; Romano, A. Covid-19

Vaccine Passport and International Traveling: The Combined Effect of Two Nudges on Americans' Support for the Pass. Journal Not Specified 2021, 1, 0. https://doi.org/

Received:

Accepted:

Published:

Publisher's Note: MDPI stays neutral with regard to jurisdictional claims in published maps and institutional affiliations.

Copyright: () 2021 by the authors Submitted to Journal Not Specified for possible open access publication under the terms and conditions of the Creative Commons Attribution (CC BY) license (https:// creativecommons.org/licenses/by/ $4.0 /)$.
1 Abstract: Immunity passports have the potential to allow large scale international traveling to resume. However, they can only become an effective tool if they are widely supported by the general public. We carry out a double blind randomized online experiment with a sample of $N=4000$ Americans to study: $i$ ) whether two nudges can increase the level of support for a COVID pass for international traveling; ii) the relationship between the effects of the nudges; and iii) if these nudges have a negative spillover on the intention to get vaccinated. We find that both nudges increase the support for the COVID pass and that their impact is stronger when they are used together. Moreover, we find that the two nudges do not affect negatively intentions to get vaccinated. Our findings have important implications for policymakers and for the nascent literature on the interaction among multiple nudges.

Keywords: COVID-19, COVID-19 Vaccine, Vaccine Passport, Nudges, Interaction Between Nudges, Peer Effect, Status Quo Bias

\section{1. Introduction}

The coronavirus disease 2019 (COVID-19) pandemic has caused catastrophic losses both in terms of human lives [1] and to the economy [2,3]. Some of the sectors that were hit more dramatically are those connected with international traveling. For instance, the tourism sector and the airline industry have been brought to their knees by the many traveling restrictions adopted worldwide [4], and by the obvious reluctance of many to travel during a pandemic [5]. According to the The World Travel and Tourism Council, in 2020 the travel and tourism sector experienced staggering losses for $\$ 3.8$ trillions [6]. Moreover, a new report of the UN's Air Transportation Agency indicates that COVID-19 caused a decline in international air travel of around $60 \%$, which resulted in losses for over $\$ 370$ billion to the airline industry [7]. However, as existing vaccines have proven to be safe and effective [8,9], these key industries are looking forward to better times, especially in light of the possible introduction of COVID-19 vaccine passports (hereinafter, COVID passes). COVID-19 passes allow the bearer to show information on their immunization status and permit people who have vaccinated against COVID-19, or have recently recovered from the virus, to face less restrictions while traveling.

While COVID passes present significant ethical and scientific challenges [10], requiring proof of vaccination for international traveling is not a new practice. For instance, the World Health Organization (WHO) has long endorsed certificates confirming vaccination against yellow fever to travel to certain countries [11]. Building on this precedent, policymakers and private companies have either introduced, or are planning to introduce, some form of COVID pass for international traveling. For example, the European Commission has reached an agreement on the "Digital COVID Certificate", which will 
provide a proof that a person has been vaccinated against COVID-19, received a negative test result, or recently recovered from COVID-19 [12]. Moreover, leading airlines are working on the IATA Travel Pass App and are considering allowing only holders of such pass to board their flights [13].

In order to be successful, COVID passes need to be widely supported by the public. From this perspective, the demise of Contact Tracing Apps is a case in point [14], showing that even the most promising and hyped technological innovation can fail to deliver if it is perceived negatively by potential adopters. In a similar vein, if COVID passes are implemented passes without the support of the general public they might lead to significant problems like lowering vaccine uptake [15]. Luckily, at least for international traveling, COVID passes seem to be perceived somewhat favourably among the general public. A recent survey carried out in the U.S. reveals that only about one third of Americans is against requiring proof of vaccination for international traveling in the form of a COVID pass [16], while in a study carried out by IATA $80 \%$ of respondents stated that they intend to use the IATA Travel Pass App as soon as it becomes available [17].

Due to their enormous potential impact, COVID passes have been hotly discussed in the academic literature [18-21]. However, all existing studies have been purely qualitative or observational [22], with one notable exception [23]. Recent research related to COVID-19 has shown that behavioral interventions can have a significant impact on people's perception of the pandemic and foster people's preventive and pro-social behaviors [24-28]. This literature has shown that experimental studies can offer precious guidance to policymakers and companies. This paper expands this literature and offers guidance on how to increase the support for a COVID pass through a communication campaign based on nudging.

In particular, we present the results from a double blind randomized online experiment with a sample of $N=4000$ Americans to test whether: $i$ ) two nudges can increase the level of support for a COVID pass for international traveling; $i$ ) there are synergies between the effects of the two nudges; and iii) these nudges generate negative spillovers on intentions to get vaccinated.

The first nudge exploits the status quo bias, which is an effective technique to increase the acceptance of a policy by presenting it as a sign of continuity with the past, and has proven effective in a variety of contexts [29]. Here, we flag that proof of vaccination for international traveling is not a novel idea, and hypothesize that this will increase the support for the COVID pass. The second nudge, instead, builds on peer effect. There is evidence that the information on peer's actions can induce pro-social behaviors [30,31], and that people tend to conform to the policy preferences of their peers [32]. Moreover, previous literature has shown a tendency to conform driven by a need to belong to a group, and the influence that the group's opinion has on the individual $[33,34]$. In this vein, we hypothesize that informing respondents about the limited opposition to COVID passes for international traveling would increase the support for this policy.

\section{Background Literature and Theoretical Framework}

Nudges are now widely accepted as an effective tool to influence behaviors without constraining individuals' ability to choose [35-37]. Recent studies have shown that nudges are effective even in the context of COVID to promote pro-social behaviors [25-27]. Here, we investigate whether they can also be used to increase the support for the COVID pass.

\subsection{Status Quo Bias}

The first nudge on which we rely is the status quo bias. The basic idea is that people are more likely to support a policy if it is perceived as a continuation of the past. In other words, whenever "an advertiser, political actor, or any other persuader wishes to make a 


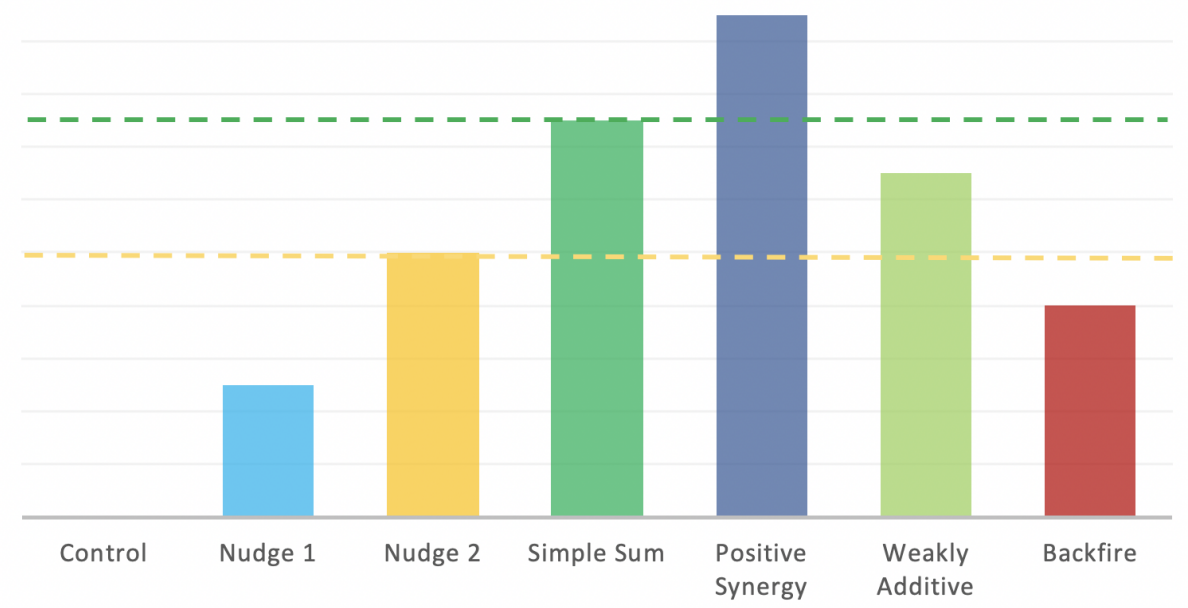

Figure 1. Possible interactions among nudges

practice or product acceptable, framing their preferred alternative as the status quo is likely to enhance its position and increase its support" [38]. For instance, a recent study shows that support for carbon mitigation policies is higher if they are presented as a continuation of the status quo [39]. Similar results are obtained even for practices as controversial as torture. Crandall et al. [38] observe that when torture is presented as a longstanding practice it is perceived as more justifiable and effective. Scholars have advanced a wide array of explanations for the existence of this bias ranging from loss aversion and regret avoidance to repeated exposure [40]. However, there is evidence that the bias also stems from people's assumption of goodness associated with the mere existence and longevity of a given state of the world [40]. Against this background, it is reasonable to expect that the status quo bias can be exploited in connection with the COVID pass. In particular, flagging that requiring proof of vaccination for international traveling is not unprecedented should trigger the status quo bias, and hence increase the support for the COVID pass.

\subsection{Peer Effect}

The second nudge we test is peer effect. Several studies found that social norms and peer influence can shape behaviors and attitudes [30,31]. In a similar vein, other studies find that polls results can influence individual level attitudes, triggering the so-called "bandwagon effect" [32]. When polls indicate that a policy is widely supported, even more people will be persuaded to support that policy [41]. Thus, polls not only describe public opinion, but can also influence it. In fact, in many democratic countries there are restrictions on carrying out and publishing polls before the elections [42]. In the context of policy support, the bandwagon effect causes an increase in support for a given policy motivated by the popularity of the policy itself. As the COVID pass for international traveling already shows a relatively good level of support among segments of the public, we attempt to leverage the bandwagon effect to further increase the support for the pass.

\subsection{Interaction Among Nudges}

Policymakers are increasingly relying on nudges to promote certain behaviors. Therefore, a key question is how multiple nudges targeted at promoting a given behavior interact. Consider the case in which nudges A and B are both effective in fostering behavior X. What happens when they are used simultaneously on the same target behavior?

As indicated in Figure 1, the interaction between two nudges can: $i$ ) be synergistic, when their joint effect is larger than the sum of the effects of each nudge separately, ii) be weakly additive, when their joint effect is larger than the effect of each of the 
two nudges when used separately, but smaller than their sum iii) backfire, when the two nudges together produce a smaller effect than either of the two nudges used alone. Understanding the kind of interaction between nudges is extremely important, as policymakers generally employ a portfolio of tools and nudges to achieve a given goal [43]. The evidence produced by the literature is, however, very limited. A recent study focused on the interaction between a moral suasion nudge aimed at reducing electricity consumption during peak load events and peer effect comparison targeting aggregate household electricity consumption [44]. The study identified a synergistic relationship between the two nudges.

A related nascent literature has attempted to identify the relationship between price incentives and nudges [43]. The handful of works carried out in this domain have produced conflicting evidence [45-47]. A recent survey on the issue concluded that due to the very small number of works on this area "our understanding ... is limited, as studies rarely use an approach that allows properly assessing synergy or the causes and mechanisms of it". [43]. One hypothesis that has been advanced is that there might be diminishing marginal returns from policy pressure, and therefore the relationship among nudges might depend on the level of support for a given policy $[43,44]$. Thus, for example, two nudges might be in a synergistic relationship when a policy has a low level of support, but then change the interaction to weakly additive when the support for the policy grows beyond a certain threshold.

We attempt to contribute to this nascent and important field of research on nudges interactions by investigating the relationship between two widely adopted nudges in a highly salient domain.

\subsection{Behavioral Spillovers}

Another important question is whether a nudge has significant spillovers that affect other activities. That is, if nudge $\mathrm{A}$, intended to promote behavior $X$, also affects behavior $Y$. The literature has found evidence of both positive and negative spillovers in different domains. For instance, a study found a strong interdependence between fuel-efficient driving styles and willingness to reduce meat consumption [48]. One reason behind positive spillovers of this kind might be that people wish to perceive themselves (and to be perceived) as consistent, and hence attempt to act in a consistent manner across different domains. In this vein, if they engage in the pro-environmental behavior $X$, they are more likely to engage in the pro-environmental behavior $Y$ [49]. Instead, in other cases scholars have observed negative spillovers. For example, a study observed that the owners of electric cars felt less compelled to engage in pro-environmental behavior than owners of traditional cars [50]. One of the possible causes of negative spillovers is the so-called moral-licensing effect. If people feel that they have done their part, they are more likely to engage in negative behaviors.

Understanding the sign of the spillovers is crucial, because in presence of negative spillovers nudges that appear to be effective might backfire by triggering a negative response on other behaviors. Scholars are therefore trying to devise experiments that investigate the existence of such spillovers and their sign. However, the debate is still ongoing and most studies so far have focused on pro-environmental behaviors. Understanding whether there are spillovers from the introduction of COVID passes and their direction should be a key priority for policymakers. For instance, if promoting the COVID pass results in lower vaccination uptake, governments should be very careful before implementing this tool.

\section{Materials and Methods}

We recruited a sample of $N=4000$ Americans on Prolific.co. To be eligible, people had to be at least 18 years of age and be resident in the U.S.. Participants took on average approximately 5 minutes and a half to complete the survey, and they were paid $\$ 0.55$. The data collection started and finished on the 15th of May 2021. We obtained informed 


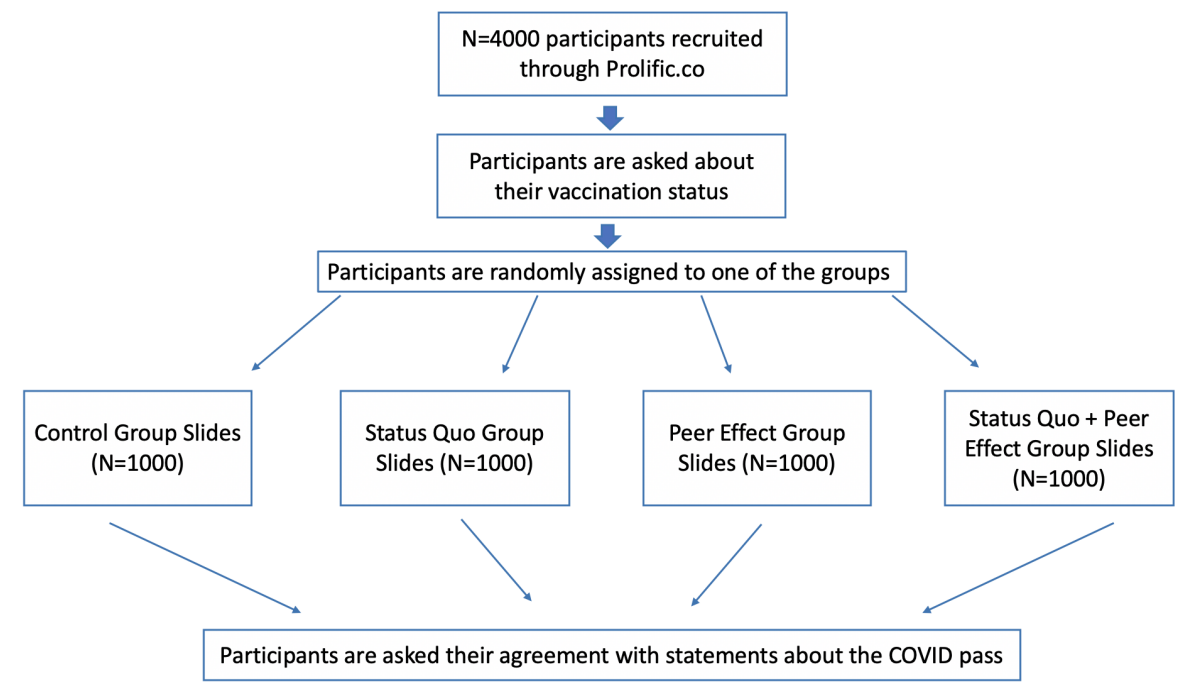

Figure 2. Experiment Flow

consent from all participants prior to the beginning of the online survey, which was approved by the faculty ethics committees of Yale University, Bocconi University and the London School of Economics.

To begin with, we asked respondents about their vaccination status, which allowed us to distinguish between respondents who completed their vaccination schedule and those who are still waiting for their first or second dose. Moreover, we asked respondents who have not yet completed their cycle whether they intend to complete their cycle or not. In the same vein, we asked respondents who have not received a vaccine whether they intend to get vaccinated when they will have the opportunity.

After this, respondents were randomly assigned to one of four different groups: Control, Status Quo, Peer Effect and Status Quo + Peer Effect (Figure 2).

The respondents in the Control Group only received basic information on the features and the purpose of a COVID pass for international traveling. The respondents included in the Status Quo condition were also informed that requiring proof of vaccination for international traveling is not unprecedented. Moreover, they were shown a picture of the International Certificate of Vaccination or Prophylaxis, or more simply the Yellow Card, endorsed by the World Health Organization to allow travelers to show proof of vaccination against yellow fever when entering certain countries. Finally, respondents in the Peer Effect condition were informed that according to a recent survey by YouGov and the Economist only one third of Americans oppose a COVID pass for international traveling. Last, respondents in the Status Quo + Peer Effect condition were informed about both the fact that requiring proof of vaccination is not a novel idea, and that only one third of Americans oppose a COVID pass for international traveling (see Figure 3).

After seeing the treatment, respondents are asked to state their level of agreements on a scale from zero to ten with statements intended to capture their support for the COVID pass. The first three statements aim at capturing the perceived importance of the COVID pass. Statements 4-8 intend to capture the perceived unfairness of the COVID pass along various dimensions. Statements 9 and 10 target additional concerns connected with the COVID pass, namely whether it can be forged and how it would affect vaccination rates. Statement 11 asks respondents whether they think that only people with a COVID pass should be allowed to board on international flights. Statement 12 deals with the troublesome finding of the literature that some people might intentionally get infected with COVID-19 if a COVID pass is introduced [51], while statement 13 intends to capture the overall perceived balance among pros and cons of the COVID pass. Table ?? reports the full list of statements, their number and the summary statistics 


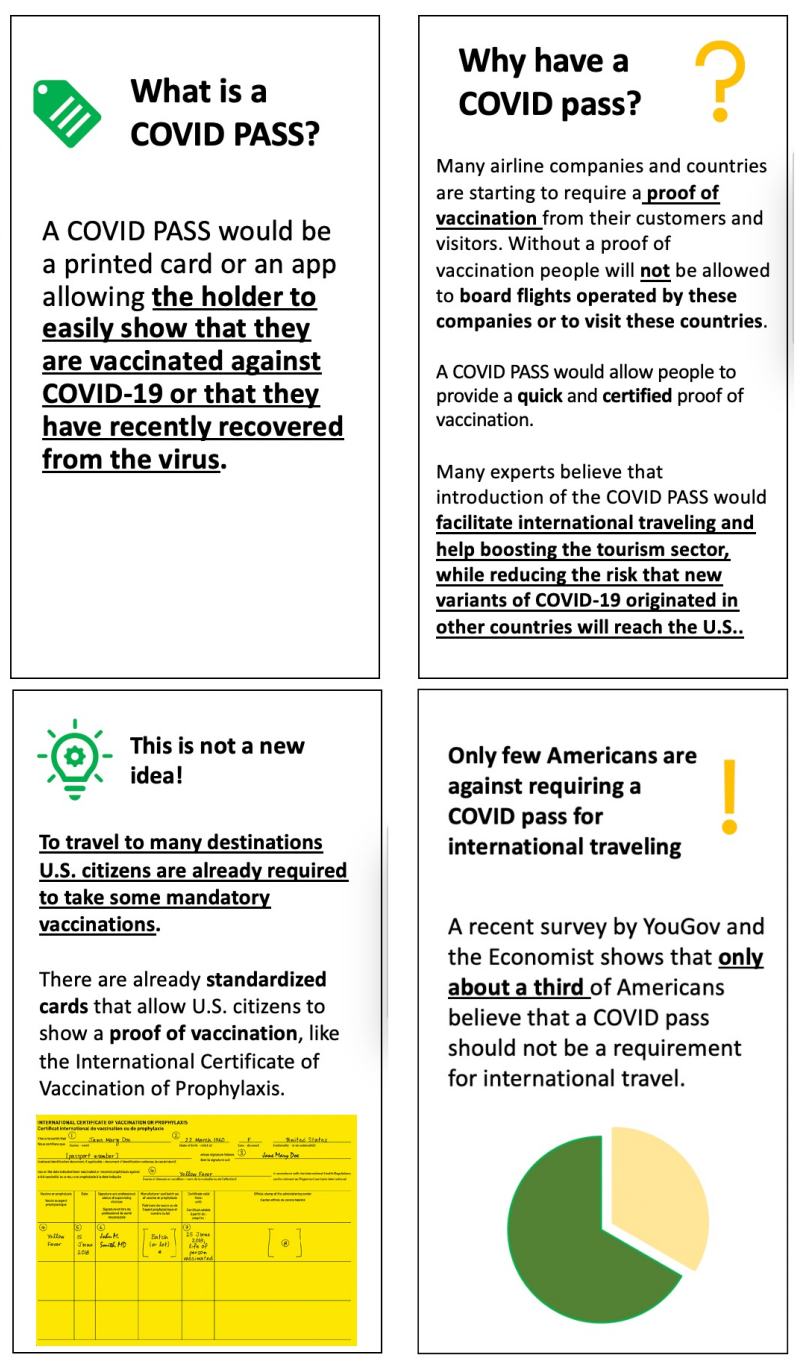

Figure 3. The top slides are shown to all participants. Respondents in the Control Group only see the top slides. Respondents in the Status Quo Group also see the bottom left slide. Respondents in the Peer Effect Group also see the bottom right slide. Respondents in the Status Quo + Peer Effect Group see all four slides. 
(mean and standard deviation). The number of the statements conveys the order in which they were asked, and will be used to denote the statements throughout the article.

Moreover, we ask respondents how likely they are to get vaccinated if a COVID pass for international traveling is introduced. The precise wording of the question depends on the vaccination status declared by the respondents at the begin of the survey. Therefore, for instance, respondents who have received only one dose are asked if they want to complete their vaccination cycle if the COVID pass is introduced. Instead, respondents who have not received any dose are asked if they intend to get vaccinated if a COVID pass is introduced. The answers are presented on a five-point Likert scale ranging from "Very unlikely" to "Very likely".

Last, we ask a series of questions that are used as controls. Such questions capture the level of trust in key institutions like the Federal Government, the respondent's State Government and the Centers for Disease Control and Prevention (CDC) or in pharmaceutical companies and tech giants. Respondents also face an attention check towards the end of the questionnaire. In the attention check respondents receive a multiple choice question in which they are asked to select the answer " 5 " if they are paying attention. The results we present in the next section exclude from the analysis the five participants who did not pass the attention check. However, the results remain robust even including these participants. We conclude the survey by asking standard demographic questions like age, education, political affiliation, etc.

\section{Results}

In this section we present the results of our experiment for each statement individually. In the Appendix, we present the results in a more concise form using factor analysis, grouping all the statements capturing the respondents' support for the pass. The two approaches produce consistent results.

The demographics of our sample are similar to U.S. demographics in many key aspects. For instance, roughly 76 percent of the U.S. population is white [52] and in our sample white respondents account for 74 percent of the participants. Our sample also closely matches the general U.S. population with respect to income distribution, percentage of republicans and education levels [52]. However, there are some differences in the composition of our sample and that of the US population. For example, $58 \%$ of our sample is composed by females, whereas the percentage of females in US is about $51 \%$ [53]. Our sample is also younger than the general population, but well represented in all the age groups. Most importantly, the sample is balanced among conditions, allowing for a clear comparison between the control and treatment groups.

\subsection{Status Quo Treatment}

We start by studying the impact of the Status Quo treatment (Table 3). We find that respondents included in this group agree more with the three statements capturing the importance of the pass with respect to respondents included in the control group. The difference is sizeable, significant at one percent $(p<0.001, p=0.002, p<0.001$, respectively) and robust to different sets of control variables for all three statements. When turning to unfairness, we find that respondents in the Status Quo agree less with statements 4-7 ( $p<0.001, p=0.014, p=0.004, p=0.008)$. Also in this case the difference is sizeable and robust to a battery of controls. Instead, we do not observe a significant difference with respect to statement 8 .

Additionally, we do not observe a significant impact of the Status Quo treatment on statements 9 and 12. Therefore, the Status Quo treatment does not induce people to believe that it is easy to forge a COVID pass, nor it induces people to state that they would intentionally get COVID if a pass is introduced. Similarly, the Status Quo does not lead respondents to state that more people will get vaccinated if a pass is introduced (statement 10). Additionally, with respect to the statement 11 we observe that the Status 
Table 1: Sample balance: the frequency table reports the Number, Percentage and Cumulative Percentage of respondents for the following variables: Political Orientation, Gender, Income, Education, Age, Employment and Race. Column 1 shows the distribution for the Control Group, Column 2 shows the distribution for the Status Quo Group, Column 3 shows the distribution for the Peer Effect Group, Column 4 shows the distribution for the Status Quo and Peer Effect Group ("PE + Status Quo") and Column 5 shows the overall distribution in the sample.

\begin{tabular}{|c|c|c|c|c|c|c|c|c|c|c|c|c|c|c|c|}
\hline & \multicolumn{15}{|c|}{ Group } \\
\hline & \multicolumn{3}{|c|}{ Control } & \multicolumn{3}{|c|}{ Status Quo } & \multicolumn{3}{|c|}{ Peer Effect } & \multicolumn{3}{|c|}{ PE + Status Quo } & \multicolumn{3}{|c|}{ Total } \\
\hline & No. & $\begin{array}{c}\text { Col } \\
\%\end{array}$ & $\begin{array}{l}\text { Cum } \\
\%\end{array}$ & No. & $\begin{array}{c}\mathrm{Col} \\
\%\end{array}$ & $\begin{array}{c}\text { Cum } \\
\%\end{array}$ & No. & $\begin{array}{c}\mathrm{Col} \\
\%\end{array}$ & $\underset{\%}{\mathrm{Cum}}$ & No. & $\begin{array}{c}\mathrm{Col} \\
\%\end{array}$ & $\underset{\%}{\text { Cum }}$ & No. & $\begin{array}{c}\text { Col } \\
\%\end{array}$ & $\begin{array}{c}\text { Cum } \\
\%\end{array}$ \\
\hline \multicolumn{16}{|l|}{ Political Orientation } \\
\hline Republican & 167 & 16.7 & 16.7 & 165 & 16.5 & 16.5 & 163 & 16.3 & 16.3 & 163 & 16.4 & 16.4 & 658 & 16.5 & 16.5 \\
\hline Democrat & 571 & 57.2 & 73.9 & 575 & 57.5 & 74.0 & 573 & 57.4 & 73.7 & 550 & 55.3 & 71.7 & 2269 & 56.8 & 73.3 \\
\hline Other or No Strong Preference & 261 & 26.1 & 100.0 & 260 & 26.0 & 100.0 & 263 & 26.3 & 100.0 & 282 & 28.3 & 100.0 & 1066 & 26.7 & 100.0 \\
\hline Total & 999 & 100.0 & & 1000 & 100.0 & & 999 & 100.0 & & 995 & 100.0 & & 3993 & 100.0 & \\
\hline \multicolumn{16}{|l|}{ Gender } \\
\hline Other/Prefer not to declare & 32 & 3.2 & 3.2 & 20 & 2.0 & 2.0 & 30 & 3.0 & 3.0 & 23 & 2.3 & 2.3 & 105 & 2.6 & 2.6 \\
\hline Female & 551 & 55.2 & 58.4 & 582 & 58.2 & 60.2 & 607 & 60.8 & 63.8 & 553 & 55.6 & 57.9 & 2293 & 57.4 & 60.1 \\
\hline Male & 416 & 41.6 & 100.0 & 398 & 39.8 & 100.0 & 362 & 36.2 & 100.0 & 419 & 42.1 & 100.0 & 1595 & 39.9 & 100.0 \\
\hline \multicolumn{16}{|l|}{ Income } \\
\hline Less than & 56 & 5.6 & 5.6 & 53 & 5.3 & 5.3 & 66 & 6.6 & 6.6 & 62 & 6.2 & 6.2 & 237 & 5.9 & 5.9 \\
\hline$\$ 10,000$ to $\$ 19,999$ & 63 & 6.3 & 11.9 & 64 & 6.4 & 11.7 & 62 & 6.2 & 12.8 & 87 & 8.8 & 15.0 & 276 & 6.9 & 12.9 \\
\hline$\$ 20,000$ to $\$ 29,999$ & 93 & 9.3 & 21.2 & 85 & 8.5 & 20.2 & 104 & 10.4 & 23.3 & 82 & 8.3 & 23.3 & 364 & 9.1 & 22.0 \\
\hline$\$ 30,000$ to $\$ 39,999$ & 81 & 8.1 & 29.4 & 99 & 9.9 & 30.1 & 95 & 9.5 & 32.8 & 96 & 9.7 & 32.9 & 371 & 9.3 & 31.3 \\
\hline$\$ 40,000$ to $\$ 49,999$ & 106 & 10.6 & 40.0 & 106 & 10.6 & 40.7 & 82 & 8.2 & 41.0 & 71 & 7.2 & 40.1 & 365 & 9.2 & 40.5 \\
\hline$\$ 50000$ to $\$ 59999$ & 112 & 11.2 & 51.2 & 111 & 11.1 & 51.9 & 93 & 9.3 & 50.4 & 82 & 8.3 & 48.3 & 398 & 10.0 & 50.4 \\
\hline$\$ 60,000$ to $\$ 69,999$ & 74 & 7.4 & 58.6 & 76 & 7.6 & 59.5 & 81 & 8.1 & 58.5 & 82 & 8.3 & 56.6 & 313 & 7.9 & 58.3 \\
\hline$\$ 70,000$ to $\$ 79,999$ & 85 & 8.5 & 67.1 & 66 & 6.6 & 66.1 & 75 & 7.5 & 66.0 & 90 & 9.1 & 65.7 & 316 & 7.9 & 66.2 \\
\hline$\$ 80,000$ to $\$ 89,999$ & 42 & 4.2 & 71.3 & 55 & 5.5 & 71.6 & 46 & 4.6 & 70.6 & 58 & 5.8 & 71.5 & 201 & 5.0 & 71.3 \\
\hline$\$ 80,000$ to $\$ 89,999$ & 54 & 5.4 & 76.8 & 57 & 5.7 & 77.3 & 55 & 5.5 & 76.1 & 53 & 5.3 & 76.8 & 219 & 5.5 & 76.7 \\
\hline$\$ 90,000$ to $\$ 99,999$ & 155 & 15.5 & 92.3 & 142 & 14.2 & 91.5 & 139 & 13.9 & 90.1 & 151 & 15.2 & 92.0 & 587 & 14.7 & 91.5 \\
\hline$\$ 150,000$ or more & 77 & 7.7 & 100.0 & 85 & 8.5 & 100.0 & 99 & 9.9 & 100.0 & 79 & 8.0 & 100.0 & 340 & 8.5 & 100.0 \\
\hline \multicolumn{16}{|l|}{ Education } \\
\hline Less than high sch & 8 & 0.8 & 0.8 & 7 & 0.7 & 0.7 & 14 & 1.4 & 1.4 & 7 & 0.7 & 0.7 & 36 & & 0.9 \\
\hline oma or equivalent) & 103 & 10.3 & 11.1 & 104 & 10.4 & 11.1 & 102 & 10.2 & 11.6 & 109 & 11.0 & 11.7 & 418 & 10.5 & 11.4 \\
\hline Some college & 206 & 20.6 & 31.7 & 230 & 23.0 & 34.1 & 230 & 23.0 & 34.6 & 227 & 22.8 & 34.5 & 893 & 22.4 & 33.8 \\
\hline Associate degree in college (2-year) & 93 & 9.3 & 41.0 & 84 & 8.4 & 42.5 & 109 & 10.9 & 45.5 & 111 & 11.2 & 45.7 & 397 & 9.9 & 43.7 \\
\hline Bachelor's degree in college & 400 & 40.0 & 81.1 & 373 & 37.3 & 79.9 & 347 & 34.7 & 80.3 & 371 & 37.3 & 83.0 & 1491 & 37.4 & 81.1 \\
\hline Master's degree or Professional Degree (JD, MD) & 176 & 17.6 & 98.7 & 177 & 17.7 & 97.6 & 169 & 16.9 & 97.2 & 148 & 14.9 & 97.9 & 670 & 16.8 & 97.8 \\
\hline Doctoral degree & 13 & 1.3 & 100.0 & 24 & 2.4 & 100.0 & 28 & 2.8 & 100.0 & 21 & 2.1 & 100.0 & 86 & 2.2 & 100.0 \\
\hline \multicolumn{16}{|l|}{$\overline{\text { Age }}$} \\
\hline $18-25$ years old & 253 & 25.3 & 25.3 & 250 & 25.0 & 25.0 & 218 & 21.8 & 21.8 & 240 & 24.1 & 24.1 & 961 & 24.1 & 24.1 \\
\hline $26-35$ years old & 343 & 34.3 & 59.7 & 354 & 35.4 & 60.4 & 358 & 35.8 & 57.7 & 317 & 31.9 & 56.0 & 1372 & 34.4 & 58.4 \\
\hline $36-45$ years old & 185 & 18.5 & 78.2 & 179 & 17.9 & 78.3 & 189 & 18.9 & 76.6 & 194 & 19.5 & 75.5 & 747 & 18.7 & 77.1 \\
\hline $46-55$ years old & 91 & 9.1 & 87.3 & 109 & 10.9 & 89.2 & 101 & 10.1 & 86.7 & 108 & 10.9 & 86.3 & 409 & 10.2 & 87.4 \\
\hline 56-65 years old & 64 & 6.4 & 93.7 & 50 & 5.0 & 94.2 & 61 & 6.1 & 92.8 & 73 & 7.3 & 93.7 & 248 & 6.2 & 93.6 \\
\hline $66-75$ years old & 21 & 2.1 & 95.8 & 19 & 1.9 & 96.1 & 24 & 2.4 & 95.2 & 31 & 3.1 & 96.8 & 95 & 2.4 & 96.0 \\
\hline$>75$ years old & 42 & 4.2 & 100.0 & 39 & 3.9 & 100.0 & 48 & 4.8 & 100.0 & 32 & 3.2 & 100.0 & 161 & 4.0 & 100.0 \\
\hline In full or part time employment & 659 & 66.0 & 100.0 & 642 & 64.2 & 100.0 & 649 & 65.0 & 100.0 & 643 & 64.6 & 100.0 & 2593 & 64.9 & 100.0 \\
\hline Student & 106 & 10.6 & 100.0 & 117 & 11.7 & 100.0 & 127 & 12.7 & 100.0 & 120 & 12.1 & 100.0 & 470 & 11.8 & 100.0 \\
\hline White & 741 & 74.2 & 100.0 & 761 & 76.1 & 100.0 & 730 & 73.1 & 100.0 & 709 & 71.3 & 100.0 & 2941 & $\begin{array}{l}73.7 \\
\end{array}$ & 100.0 \\
\hline
\end{tabular}




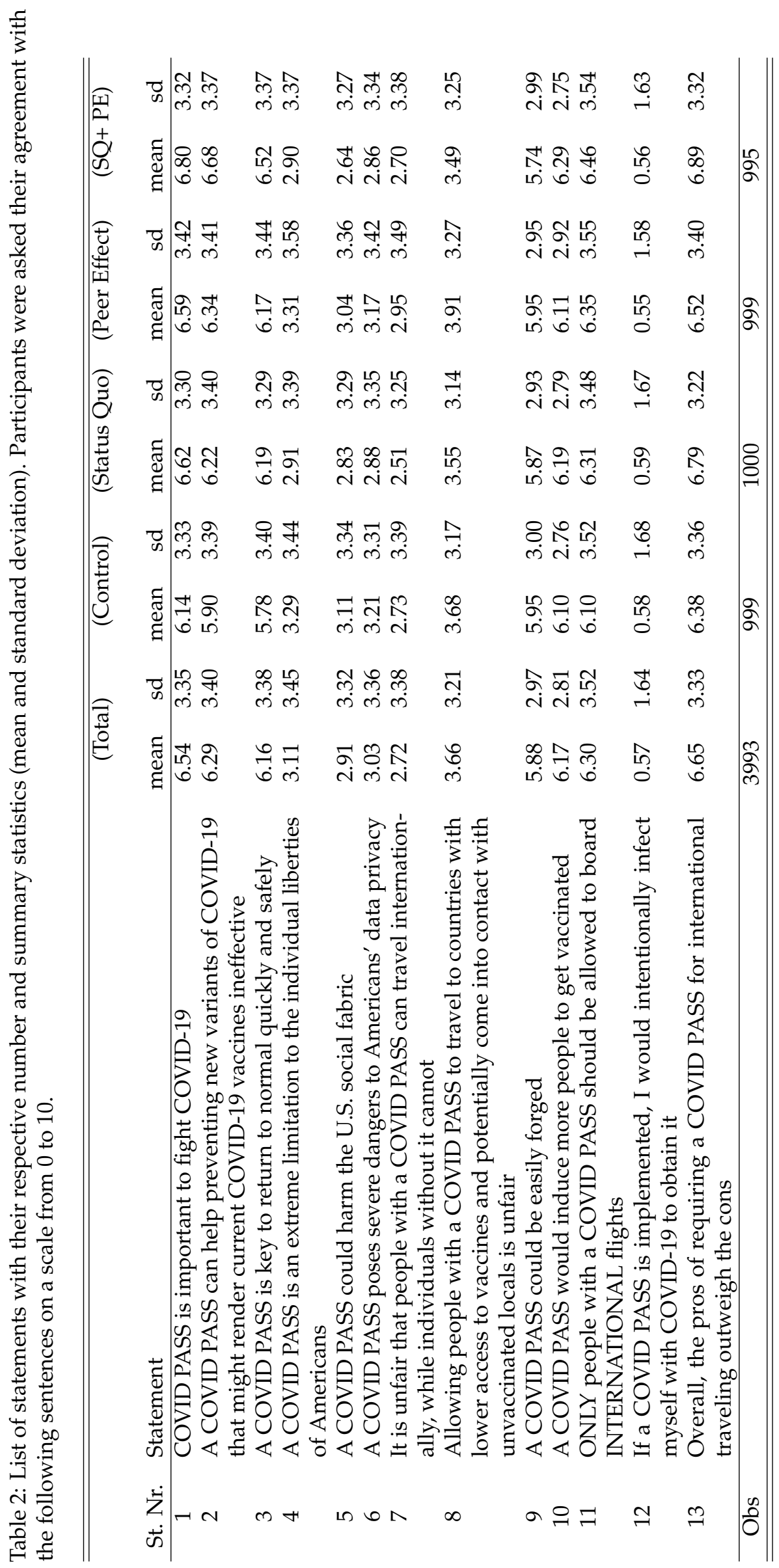


Table 3: OLS beta coefficients deriving from regressions with the statements as the dependent variable and a binary variable to measure the impact of being in the treatment groups with respect to the Control group. The regressions are ran controlling for demographics, vaccination status, frequency of travel and trust levels of the participants. Tables of the regressions output are included in the Appendix.

\begin{tabular}{cccccccc}
\hline Nr. Stmt & $\mathbf{N}$ & $\beta_{S Q}$ & $p_{S Q}$ & $\beta_{P E}$ & $p_{P E}$ & $\beta_{P E+S Q}$ & $p_{P E+S Q}$ \\
\hline 1 & 3705 & 0.525 & $<0.001$ & 0.56 & $<0.001$ & 0.707 & $<0.001$ \\
2 & 3704 & 0.404 & 0.002 & 0.544 & $<0.001$ & 0.88 & $<0.001$ \\
3 & 3696 & 0.471 & $<0.001$ & 0.48 & $<0.001$ & 0.792 & $<0.001$ \\
4 & 3656 & -0.413 & 0.001 & -0.101 & 0.438 & -0.485 & $<0.001$ \\
5 & 3639 & -0.308 & 0.014 & -0.195 & 0.128 & -0.527 & $<0.001$ \\
6 & 3636 & -0.364 & 0.004 & -0.188 & 0.137 & -0.443 & $<0.001$ \\
7 & 3634 & -0.331 & 0.008 & 0.0755 & 0.566 & -0.134 & 0.302 \\
8 & 3664 & -0.207 & 0.137 & 0.175 & 0.218 & -0.264 & 0.059 \\
9 & 3703 & -0.09 & 0.488 & -0.0582 & 0.66 & -0.221 & 0.094 \\
10 & 3701 & 0.107 & 0.362 & 0.0308 & 0.797 & 0.17 & 0.141 \\
11 & 3693 & 0.305 & 0.024 & 0.302 & 0.027 & 0.361 & 0.008 \\
12 & 3579 & 0.0314 & 0.681 & -0.0094 & 0.897 & 0.0046 & 0.951 \\
13 & 3690 & 0.494 & $<0.001$ & 0.24 & 0.055 & 0.592 & $<0.001$ \\
\hline
\end{tabular}

Quo leads respondents to agree more with the idea that only people with a COVID pass should be able to board international flights $(p=0.024)$.

Last, respondents in the Status Quo condition agree more with the statement 13 $(p<0.001)$, and therefore consider the pros of a COVID pass to outweigh its cons. Table 3 presents the results of regressions ran with the statement as a dependent variable, the treatment as the main independent variable and controls for demographics, vaccination status and trust levels of the participants. We refer the reader to the online Appendix for the full regression tables, including the results from regressions with different sets of controls.

To summarize, we conclude that the status quo bias is highly effective in increasing the perceived importance of the COVID pass (statements 1-3), in reducing its perceived unfairness (statements 4-7, with the exception of statement 8), and overall increases the support for the COVID pass (statement 13).

\section{Peer Effect Treatment}

As for the Status Quo, we find that respondents included in this group agree more with the three statements capturing the importance of the pass with respect to respondents included in the control group (see Table 3). The magnitude of the effects is large, statistically significant ( $p<0.001$ for all statements) and robust to various sets of control variables for all three statements. When turning to unfairness, instead, we observe that the Peer Effect treatment is not effective. The Peer Effect treatment does not impact the level of agreement with statement 9 and 12, and it does not lead respondents to state that more people will get vaccinated if a pass is introduced (statement 10). Last, respondents in the Peer Effect condition agree more with the idea that the the pros of a COVID pass outweigh the cons (statement 13), but the result is weaker than for the Status Quo $(p=0.55)$. Table 3 presents the results of regressions ran with the statement as a dependent variable, the treatments as the main independent variable and controls for demographics, vaccination status, frequency of travel and trust levels of the participants. We refer the reader to the online Appendix for the full regression tables, including the results from regressions with different sets of controls.

Overall, the peer effect condition has a positive and statistically significant impact on the perceived importance of the COVID pass (statements 1-3), but it has a limited impact on the perceived unfairness (statements 4, 6, 7, 8 with statement 5 being the exception). Moreover, it increases the overall support for the COVID pass (statement 13). 


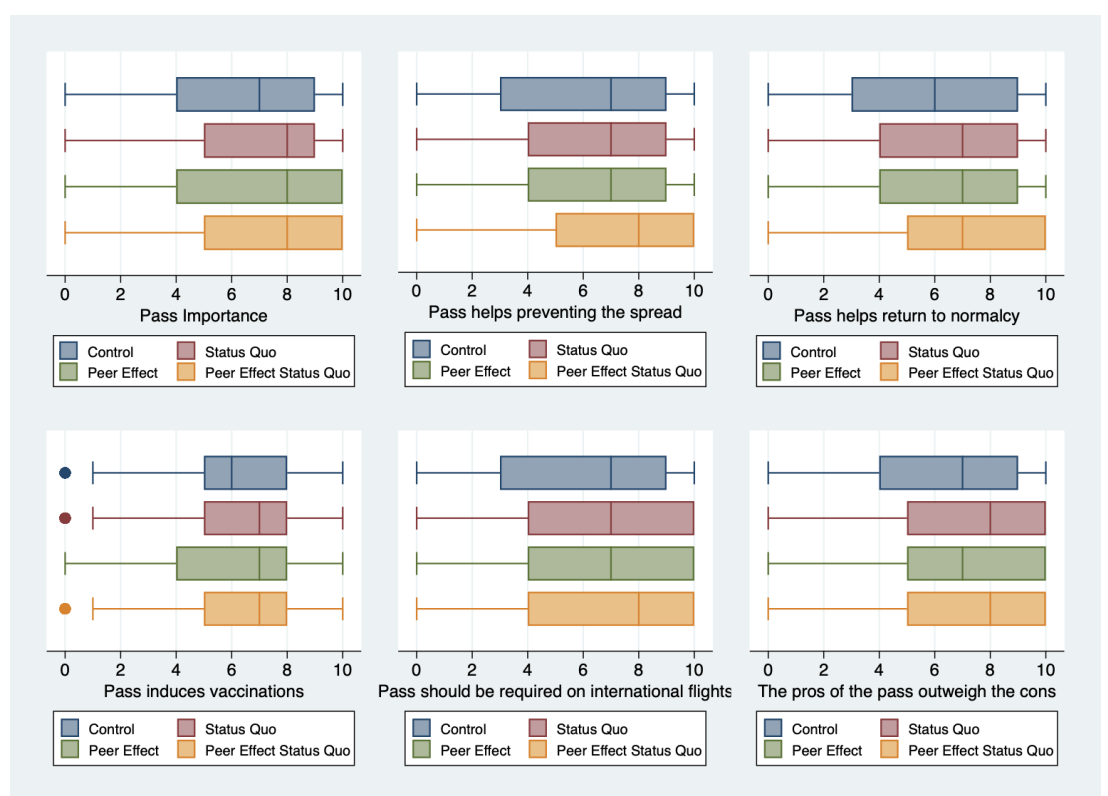

Figure 4. Summary of results for the statements meant to capture positive attributes of the COVID pass (statements 1-3, 10, 11 and 13). The boxplots show the distribution of responses in the Control, Status Quo, Peer Effect and Peer Effect and Status Quo groups without any additional control. A higher value corresponds to more favorable attitudes towards the pass.

\section{Status Quo + Peer Effect Treatment}

Then, we turn to studying the impact of the two nudges when they are used simultaneously. Starting with the statements on the importance of the pass, we find that the joint impact of the nudges is statistically significant ( $p<0.001$ for all statements) and larger than the impact of both nudges used separately (Table 3), showing weak additionality in the treatments' effects. For instance, for Statement 2 the joint impact of the two nudges is $218 \%$ larger than the effect of the Status Quo alone and and $162 \%$ larger than that of peer effect.

Combining the two nudges is also very effective in reducing the perceived level of unfairness. Respondents in this condition agree less with statements 4, 5, 6 and 8 ( $p<$ $0.001, p<0.001, p<0.001, p=0.059$ ). In line with the Status Quo and the Peer Effect conditions, for statements 9,10 and 12 we observe no significant differences between this condition and the control. Instead, we see that for statement 11 the joint impact of the two treatments is slightly larger and more statistically significant $(p=0.008)$ than that of the status quo and the peer effect when used separately.

Last, this treatment is the most effective in persuading people that the pros of the COVID pass outweigh the cons (statement 13) $(p<0.001)$.

Table 3 presents the results of regressions ran with the statement as a dependent variable, the treatment as the main independent variable and controls for demographics, vaccination status, frequency of travel and trust levels of the participants. We refer the reader to the online Appendix for the full regression tables, including the results from regressions with different sets of controls.

Overall, for virtually all statements we find that the two treatments used together have a stronger impact. Figures 4 and 5 summarizes our findings.

To further ensure that our results are robust, we carry out a post hoc test to estimate the marginal effect of the combination of the two nudges relative to the effect of each one separately. The results are included in the online Appendix and are consistent with the results reported in Table 3. 


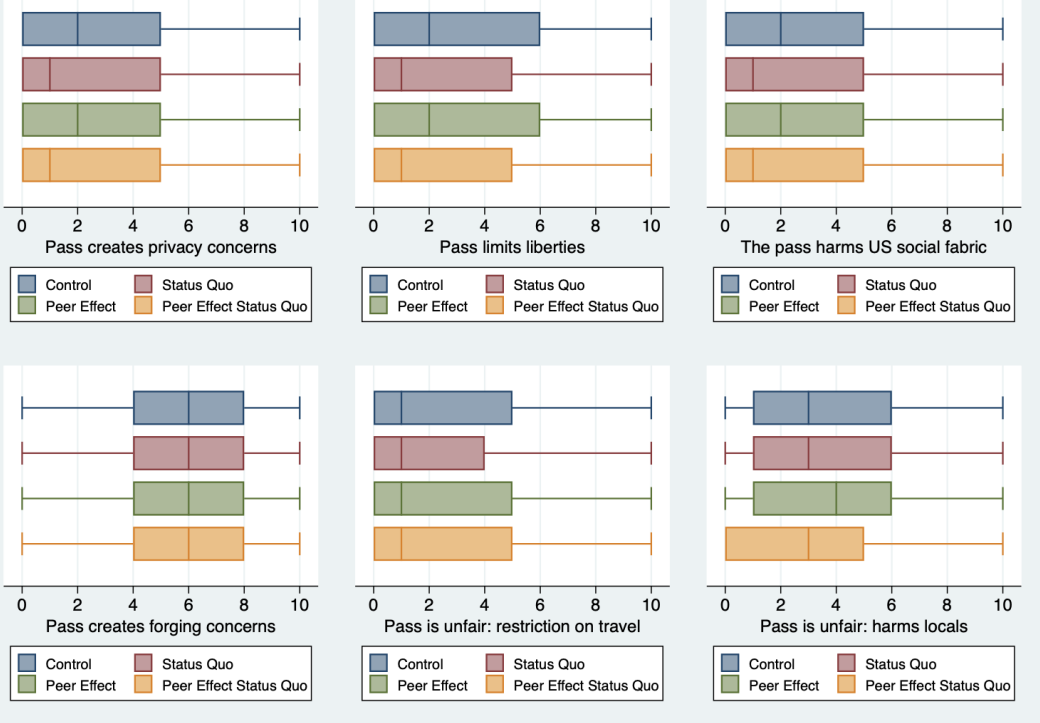

Figure 5. Summary of results for the statements meant to capture negative attributes of the COVID pass (statements 4-9). The boxplots show the distribution of responses in the Control, Status Quo, Peer Effect and Peer Effect and Status Quo groups without any additional control. A lower value corresponds to more favourable attitudes towards the pass.

Table 4: OLS beta coefficients deriving from regressions with the intention to get vaccinated as the dependent variable and a binary variable to measure the impact of being in the treatment groups with respect to the Control group. The regressions are ran controlling for demographics, vaccination status, frequency of travel and trust levels of the participants. Tables of the regressions output are included in the Appendix.

\begin{tabular}{cccccccc}
\hline Status & $\mathbf{N}$ & $\beta_{S Q}$ & $p_{S Q}$ & $\beta_{P E}$ & $p_{P E}$ & $\beta_{P E+S Q}$ & $p_{P E+S Q}$ \\
\hline Unvacinnated & 1158 & 0.0124 & 0.897 & -0.12 & 0.238 & -0.01 & 0.919 \\
One Dose & 433 & -0.275 & 0.059 & -0.0807 & 0.501 & 0.103 & 0.31 \\
Vaccinated & 2121 & -0.03 & 0.494 & 0.007 & 0.879 & -0.026 & 0.572 \\
All Sample & 3716 & -0.0535 & 0.279 & -0.07 & 0.169 & -0.033 & 0.505 \\
\hline
\end{tabular}

\section{Intention to get Vaccinated}

Last, we turn to investigate whether the treatments affect the intention to get vaccinated if a COVID pass is introduced. The results are indicated in Table 4. We carry out the analysis considering separately the respondents who have completed a cycle of vaccination, the respondents who have received only one dose, and respondents who have not received a dose yet.

We find that the treatments do not have a negative impact on the intention to get vaccinated in case a COVID pass is introduced for any of these groups. Moreover, we find that the treatments do not have a negative impact also when aggregating the three groups (Table 4).

Thus, the introduction of a COVID pass is unlikely to negatively affect intentions to get vaccinated.

\section{Discussion}

Our experiment revealed that the status quo bias and peer effect can be used together as an effective mean to increase support for the COVID pass, without reducing intention to get vaccinated. These findings have both immediate policy implications and broader theoretical implications. 


\section{Policy Implications}

COVID passes could play a crucial role in restarting large scale international traveling. However, for them to be successful they must be perceived as both important and fair by the general public. Our results can aid policymakers in ensuring that these conditions are met.

Flying without being vaccinated imposes significant externalities on the country of destination, the other passengers on the flight, and society at large. As noted by Sunstein [35], nudges aimed at mitigating a negative externality are not particularly controversial from an ethical perspective. The only relevant issue is whether they are effective. From this perspective, we find that both the status quo and peer effect are highly effective in increasing the support for the COVID pass. Most importantly, our results suggest that policymakers who want to increase the support for the COVID pass should rely on the two nudges simultaneously, as this allows them to both increase the perceived importance and the perceived fairness of the COVID pass. In fact, while the relationship between the nudges is not synergistic but weakly additive, the cost of each nudge is likely to be minimal and their combined effect is larger. However, so policymakers often neglected to flag that requiring proof of vaccination for international traveling is not unprecedented, and that in the case of COVID it would be opposed by only a minority of people (e.g., [54]).

Nevertheless, in some contexts policymakers and private companies might not have the possibility to employ both nudges. For instance, according to the Advertising Research Foundation (ARF) 6 seconds TV advertising are very effective in commanding more attention per second than longer advertisements [55]. For such short communications, policymakers and private companies might have to focus on a single nudge. In this case, if they intend to emphasize the importance of the COVID pass they should rely on the peer effect nudge, whereas if they intend to flag the fairness of the pass they should build on the status quo bias.

\section{Theoretical Implications}

At a theoretical level, our work contributes to two strands of literature that are gaining momentum: (i) the study of interactions among nudges; and (ii) behavioral spillovers triggered by nudges.

The literature on interaction among nudges is in its infancy and, to the best of our knowledge, there are no studies testing the interaction between peer effect and the status quo bias, especially in health-related domains. As these nudges are among the most widely studied in the literature and are effective in many domains, it is important to understand whether they can be used together or if their joint use is likely to backfire. Our results reveal that at least in relation to COVID vaccine pass, the status quo and the peer effect nudges are effective together and their effects are weakly additive.

While our results refer to a very unique setting, there are reasons to believe that the status quo bias and the peer effect could often be in a weakly additive relationship. First, by definition, a peer effect nudge as the one used in our experiment can be implemented only for policies that already have a fair amount of support. Therefore, it is likely that for these policies the marginal returns from policy pressure are diminishing at a fairly fast rate. This argument holds both at society level, as greater support implies that there are only a few people left to convince, and at the individual level, where support is already high and cannot further increase by much. This might make it unlikely to observe a synergistic relationship when using the status quo in combination with other nudges nudges.

Second, while they exploit different mechanisms, there is a connection between the two forms of nudges, as they both rely on social influence. Social psychology distinguishes between two main forms of social influence: informational social influence (telling people what is commonly done) and normative social influence (informing them about what is widely approved) [33,34]. The nudges we implemented in this paper are 
likely to stimulate both forms of social influence. In fact, the status quo bias suggests that in the past there was sufficient support for the policy that it was implemented, whereas the peer effect suggests that today there is widespread support for the policy in question. The interaction of informational and normative social influence implicitly pushes the individual in the same direction, making it reasonable to expect some compounding in the effects of the two nudges.

Moreover, as neither on the two interventions relies on monetary incentives, it is unlikely that extrinsic motivations would crowd out the intrinsic motivations triggered by the nudges. For these reasons, we suggest that combining status quo and peer effect is highly unlikely to backfire.

In addition we extend the literature on behavioral spillovers by investigating whether nudges on highly topical issues can generate a backlash on other key behaviors. We observe that in this context neither of the treatments had a negative impact on the intention to get vaccinated, thus suggesting that negative spillovers are not going to offset the positive impact of these nudges.

\section{Limitations of the Analysis and Future Research}

From a methodological standpoint, our study suffers from two limitations. First, we carry out an online experiment, and hence - like with every online experiment one can question its external validity. This is partially alleviated by the heterogeneous composition of our sample and its large size. Second, while we rely on a large sample that closely matches the U.S. population along key variables, our sample is not representative. However, online experiments with non-representative samples are widely used across many disciplines and have been proven to be a reliable source of information, with a good degree of generalizability [56]. Moreover, the magnitude of the coefficients is large and robust to different sets of controls, suggesting that our results are likely to be informative even if the sample is not representative.

Additionally, the effect of our treatments is also likely to be context dependent. For instance, it is possible that the impact of peer effect treatment might change, once the percentage of people opposing the COVID pass changes. Therefore, it is of key importance to implement these nudges before too many people start opposing COVID pass. Last, we do not include a manipulation check in our experiment.

Author Contributions: CS and AR had the idea, CS, MA, RR and AR did the literature search, and were involved in the design of the online experiment. CS conducted the analysis of the data. CS and AR created the tables and wrote the first draft of the manuscript. All authors provided critical feedback on the draft and reviewed and approved the final version for publication.

\section{Funding: This research received no external funding}

Institutional Review Board Statement: The study was approvide by the Institutional Review Board of Yale University, Bocconi University and the London School of Economics. Institutional Review Board of Yale University (nr. 2000030495):This research was deemed exempt under 45CFR46.104 (2)(ii). Institutional Review Board of Bocconi University (nr. SA000294): we deem your research project "The Impact of Two Nudges on COVID-19 Vaccine Passport Acceptance" SA000294 to be compliant with the Research Ethics Policy of the University. Ethical Review Board of the London School of Economics (nr. 23637 original approval): Approved as low risk project.

Informed Consent Statement: Informed consent was obtained from all subjects involved in the study

Data Availability Statement: The data to replicate the analysis will be made publicly available. The anonymized responses of the survey participants will be made available by the author to who requests such data.

Acknowledgments: We thank Ian Ayres and Tom Tyler for their helpful comments. All errors are ours.

Conflicts of Interest: The authors declare no conflict of interest 
1. WHO. WHO Coronavirus (COVID-19) Dashboard. Online at https: / / covid19.who.int, 2021.

2. McKibbin, W.; Fernando, R. The economic impact of COVID-19. Economics in the Time of COVID-19 2020, 45.

3. Cutler, D.M.; Summers, L.H. The COVID-19 pandemic and the $\$ 16$ trillion virus. Jama 2020, 324, 1495-1496.

4. Gössling, S.; Scott, D.; Hall, C.M. Pandemics, tourism and global change: a rapid assessment of COVID-19. Journal of Sustainable Tourism 2020, 29, 1-20.

5. Immordino, G.; Jappelli, T.; Oliviero, T.; Zazzaro, A. Fear of COVID-19 Contagion and Consumption: Evidence from a Survey of Italian Households. Technical report, Centre for Studies in Economics and Finance (CSEF), University of Naples, Italy, 2021.

6. Schengenvisainfo. WTTC: Global Travel Tourism Sector Lost Around €3,8 Trillion in 2020 Due to COVID-19. Online at https:/ / www.schengenvisainfo.com/news/wttc-global-traveltourism-sector-lost-around-e38-trillion-in-2020-due-to-covid-19/, 2021.

7. UN-News. Air travel down 60 per cent, as airline industry losses top $\$ 370$ billion: ICAO. Online at https://news.un.org/en/story/2021/01/1082302, 2021.

8. Prü $\beta$, B.M. Current State of the First COVID-19 Vaccines. Vaccines 2021, 9, 30.

9. Haas, E.J.; Angulo, F.J.; McLaughlin, J.M.; Anis, E.; Singer, S.R.; Khan, F.; Brooks, N.; Smaja, M.; Mircus, G.; Pan, K.; others. Impact and effectiveness of mRNA BNT162b2 vaccine against SARS-CoV-2 infections and COVID-19 cases, hospitalisations, and deaths following a nationwide vaccination campaign in Israel: an observational study using national surveillance data. The Lancet 2021.

10. Hall, M.A.; Studdert, D.M. "Vaccine Passport" Certification-Policy and Ethical Considerations. New England Journal of Medicine 2021.

11. UN-News. Amendment to International Health Regulations, 2005, annex 7 (yellow fever). Online at https: / / www.who.int/ith/annex7-ihr.pdf2, 2013.

12. EuropeanCommission. EU Digital COVID Certificate: European Parliament and Council reach agreement on Commission proposal. Online at https: / / ec.europa.eu/commission/ presscorner/detail/en/IP_21_2593, 2021.

13. IATA. IATA Travel Pass Initiative. Online at https://www.iata.org/en/programs/ passenger/travel-pass/, 2021.

14. Rowe, F.; Ngwenyama, O.; Richet, J.L. Contact-tracing apps and alienation in the age of COVID-19. European Journal of Information Systems 2020, 29, 545-562.

15. NBC. Politicization of 'vaccine passports' could aggravate GOP hesitancy, experts warn. Online at https:/ / www.nbcnews.com/politics/politics-news / politicization-vaccine-passportscould-aggravate-gop-hesitancy-experts-warn-n1263048, 2021.

16. YouGovAmerica. Americans tend to support vaccine passports for international travel. https:/ /today.yougov.com/topics/travel/articles-reports/2021/04/21/americans-tendsupport-vaccine-passports-internati, 2021.

17. IATA. Travelers Gaining Confidence. Online at https:/ /www.iata.org/en/pressroom/pr/ 2021-03-09-01/, 2021.

18. Osama, T.; Razai, M.S.; Majeed, A. Covid-19 vaccine passports: access, equity, and ethics, 2021.

19. Memish, Z.A.; Alharthy, A.; Alqahtani, S.A.; Karakitsos, D. COVID-19 air travel restrictions and vaccine passports: An ongoing debate. Travel Medicine and Infectious Disease 2021.

20. Tanner, R.; Flood, C.M. Vaccine Passports Done Equitably. JAMA Health Forum. American Medical Association, 2021, Vol. 2, pp. e210972-e210972.

21. Pavli, A.; Maltezou, H.C. COVID-19 vaccine passport for a safe resumption of travel. Journal of Travel Medicine 2021.

22. Drury, J.; Mao, G.; John, A.; Kamal, A.; Rubin, J.; Stott, C.; Vandrevala, T.; Marteau, T. Behavioural responses to Covid-19 health certification: A rapid review. medRxiv 2021.

23. Guidi, S.; Romano, A.; Sotis, C. Depolarizing the COVID-19 Vaccine Passport. Yale Law Journal (Forum) 2021, SSRN: https://ssrn.com/abstract=3850152, http://dx.doi.org/10.2139/ssrn.3850152.

24. Romano, A.; Sotis, C.; Dominioni, G.; Guidi, S. The scale of COVID-19 graphs affects understanding, attitudes, and policy preferences. Health economics 2020, 29, 1482-1494.

25. Pennycook, G.; McPhetres, J.; Zhang, Y.; Lu, J.G.; Rand, D.G. Fighting COVID-19 misinformation on social media: Experimental evidence for a scalable accuracy-nudge intervention. Psychological science 2020, 31, 770-780. 
26. Kim, J.; Giroux, M.; Gonzalez-Jimenez, H.; Jang, S.; Kim, S.; Park, J.; Kim, J.E.; Lee, J.C.; Choi, Y.K. Nudging to reduce the perceived threat of Coronavirus and stockpiling intention. Journal of Advertising 2020, pp. 1-15.

27. Milkman, K.L.; Patel, M.S.; Gandhi, L.; Graci, H.N.; Gromet, D.M.; Ho, H.; Kay, J.S.; Lee, T.W.; Akinola, M.; Beshears, J.; others. A megastudy of text-based nudges encouraging patients to get vaccinated at an upcoming doctor's appointment. Proceedings of the National Academy of Sciences 2021, 118.

28. Ceylan, M.; Hayran, C. Message Framing Effects on Individuals' Social Distancing and Helping Behavior During the COVID-19 Pandemic. Frontiers in psychology 2021, 12, 663.

29. Samuelson, W.; Zeckhauser, R. Status quo bias in decision making. Journal of risk and uncertainty 1988, 1, 7-59.

30. Allcott, H. Social norms and energy conservation. Journal of public Economics 2011, 95, 10821095.

31. Ayres, I.; Raseman, S.; Shih, A. Evidence from two large field experiments that peer comparison feedback can reduce residential energy usage. The Journal of Law, Economics, and Organization 2013, 29, 992-1022.

32. Rothschild, D.; Malhotra, N. Are public opinion polls self-fulfilling prophecies? Research $\mathcal{E}$ Politics 2014, 1, 2053168014547667.

33. Fiske, S.T. Social beings: Core motives in social psychology; John Wiley \& Sons, 2018.

34. Deutsch, M.; Gerard, H.B. A study of normative and informational social influences upon individual judgment. The journal of abnormal and social psychology 1955, 51, 629.

35. Sunstein, C.R. The ethics of nudging. Yale J. on Reg. 2015, 32, 413.

36. Loewenstein, G.; Chater, N. Putting nudges in perspective. Behavioural Public Policy 2017, $1,26$.

37. Romano, A.; Sotis, C. Odi et Amo: A nudge to reduce the consumption of single-use carrier bags. Waste Management 2021, 120, 382-391.

38. Crandall, C.S.; Eidelman, S.; Skitka, L.J.; Morgan, G.S. Status quo framing increases support for torture. Social Influence 2009, 4, 1-10.

39. Lang, C.; Weir, M.; Pearson-Merkowitz, S. Status quo bias and public policy: evidence in the context of carbon mitigation. Environmental Research Letters 2021, 16, 054076.

40. Eidelman, S.; Crandall, C.S. Bias in favor of the status quo. Social and Personality Psychology Compass 2012, 6, 270-281.

41. Hardmeier, S. The effects of published polls on citizens. The SAGE handbook of public opinion research 2008, pp. 504-513.

42. Barnfield, M. Think twice before jumping on the bandwagon: Clarifying concepts in research on the bandwagon effect. Political Studies Review 2020, 18, 553-574.

43. Drews, S.; Exadaktylos, F.; van den Bergh, J.C. Assessing synergy of incentives and nudges in the energy policy mix. Energy Policy 2020, 144, 111605.

44. Brandon, A.; List, J.A.; Metcalfe, R.D.; Price, M.K.; Rundhammer, F. Testing for crowd out in social nudges: Evidence from a natural field experiment in the market for electricity. Proceedings of the National Academy of Sciences 2019, 116, 5293-5298.

45. Hilton, D.; Charalambides, L.; Demarque, C.; Waroquier, L.; Raux, C. A tax can nudge: The impact of an environmentally motivated bonus/malus fiscal system on transport preferences. Journal of Economic Psychology 2014, 42, 17-27.

46. Schall, D.L.; Wolf, M.; Mohnen, A. Do effects of theoretical training and rewards for energyefficient behavior persist over time and interact? A natural field experiment on eco-driving in a company fleet. Energy Policy 2016, 97, 291-300.

47. Panzone, L.A.; Ulph, A.; Zizzo, D.J.; Hilton, D.; Clear, A. The impact of environmental recall and carbon taxation on the carbon footprint of supermarket shopping. Journal of Environmental Economics and Management 2018.

48. Van der Werff, E., e.a. I am what I am, by looking past the present: the influence of biospheric values and past behavior on environmental self-identity. Environment and Behavior 2013, $42,1-32$.

49. Cialdini, R.B, e.a. Preference for consistency: the development of a valid measure and the discovery of surprising behavioral implications. Journal of personality and social psychology 1995, 42, 318-328.

50. Klockner, C.A., e.a. Positive and negative spillover effects from electric car purchase to car use. Transportation Research D: Transport and Environment 2013, 42, 32-38. 
51. Garrett, P.M.; White, J.P.; Lewandowsky, S.; Kashima, Y.; Perfors, A.; Little, D.R.; Geard, N.; Mitchell, L.; Tomko, M.; Dennis, S. The acceptability and uptake of smartphone tracking for COVID-19 in Australia. Plos one 2021, 16, e0244827.

52.

53. WorldBank. Population, female (\% of total population) - United States. Online at https: / / data.worldbank.org/indicator/SP.POP.TOTL.FE.ZS?locations=US, 2019.

54. Messaggero. Mario Draghi: «Italia avrà il suo Green pass prima di quello europeo». Online at https:/ / www.ilmessaggero.it/video/politica/draghi_italia_avra_green_pass_prima_di_ europeo-5974701.html, 2021.

55. MarketingDive. 6-second TV ads command more attention per second than traditional spots. Online at https: / / www.marketingdive.com/news / 6-second-tv-ads-command-moreattention-per-second-than-traditional-spots/525770/, 2018.

56. Mullinix, K.J.; Leeper, T.J.; Druckman, J.N.; Freese, J. The generalizability of survey experiments. Journal of Experimental Political Science 2015, 2, 109-138. 
